\title{
u-KoMIPS: A Medical Image Processing System in a Ubiquitous Environment
}

\author{
Soo Jin Lee and Moon Hae Kim \\ Konkuk University, \\ Seoul 143-170, Korea \\ \{leesj, mhkim\}@konkuk.ac.kr
}

\begin{abstract}
The ubiquitous computing paradigm has made the telemedicine field being changed. However, research on the telemedicine field in a ubiquitous environment has not been carried out yet. In this paper, we have designed and implemented a ubiquitous medical image processing system for telemedicine applications based on the time-triggered message-triggered object (TMO) structuring scheme that is a distributed real-time object model. The initial version of our system is named KoMIPS (Konkuk Medical Image Processing System) that is a result of joint work with a Samsung Medical Center team. KoMIPS is a stand-alone system. Currently, KoMIPS is being extended to run on a ubiquitous environment. The extended version, named u-KoMIPS (ubiquitous KoMIPS), is designed with the TMO model and its execution environment is based on TMOSM/Linux that is an execution engine for a TMO structured program on Linux. The u-KoMIPS can acquire a medical image from various medical image scanners (e.g., MRI, CT, gamma camera, etc.) and then convert it into a DICOM image, transfer the DICOM image to one or more clients. Then, a client can analyze, process, and diagnose the DICOM image. We expect that telemedicine applications based on our system would accurately acquire a medical image from various medical image devices and efficiently analyze it.
\end{abstract}

\section{Introduction}

Today, demands on an effective telemedicine system have been ever increasing. With the advances in information technologies and telecommunication technologies, the construction of an effective telemedicine system becomes possible. And embedded systems equipped with microprocessors are widely applied to various fields with advances in information technologies and telecommunication technologies. In addition, the ubiquitous computing paradigm leads application areas of embedded systems to become much broader. Many fields including the telemedicine field are affected by this phenomenon. Now, research and development for various medical systems is being conducted, for example, u-hospital, telemedicine used IMT 2000 service, and web-based PACS. In Europe, mobile telemedicine applications using a cellular phone or a PDA have already been introduced [1-3]. The vigorous progress in telemedicine is being made due to efficient environments such as improvement IT business, inter- 
net communication network, PACS, wireless LAN, mobile phone, etc. However, most of current telemedicine systems depend on special platform/devices and those are not compatible each other [4]. Also, research on a ubiquitous telemedicine is not in a mature stage.

In this paper, we propose a ubiquitous telemedicine system by extending KoMIPS (Konkuk Medical Image Processing System) that has been developed in collaboration with a Samsung Medical Center team. KoMIPS consists of a small gamma camera for a breast cancer and medical image processing system. We have remodeled and implemented KoMIPS to a TMO based medical image processing system for telemedicine applications. The TMO model is a distributed real-time object model and briefly introduced in section 2 .

Our extended system, named u-KoMIPS (ubiquitous KoMIPS) can acquire a medical image from various medical image devices (such as MRI, CT, and gamma camera) and then converts it into an equivalent DICOM (Digital Image and Communication in Medicine) image that is an international medical image format standard. The u-KoMIPS provides a set of analysis functions such as ROI (region of interest) setting, contrast adjust, MCA (multi-channel analyzer), and a set of image processing functions such as filtering, edge detection, and binary arithmetic.

The rest of this paper is organized as follows: In section 2, we briefly introduce the TMO model and DICOM format that are the basis of our approach. Section 3 presents KoMPIS overview and the TMO based medical image processing system. In section 4, we introduce an application that has been developed applied TMO approach. Finally, section 5 summarizes the paper with future work.

\section{Background}

\subsection{TMO Scheme}

TMO is a natural, syntactically minor, and semantically powerful extension of the conventional Object(s) [5-7]. Particularly, TMO is a high-level real-time computing object. Member functions (i.e., methods) are executed within specified time. Timing requirements are specified naturally intuitive forms with no esoteric styles imposed. As depicted in Figure 1, the basic TMO structure consists of four parts:

- Object Data Store (ODS): the basic unit of storage which can be exclusively accessed by a certain TMO method execution at any given time or shared among concurrent executions of TMO methods (SpMs or SvMs).

- Environment Access Capability (EAC): the list of entry points to remote object methods, logical communication channels, and I/O device interfaces.

- Spontaneous Methods (SpM): a new type of method, also known as the timetriggered (TT) method. The SpM executions are triggered when the real-time clock reaches specific values determined at design time. A SpM has an AAC (Autonomous Activation Condition), which is a specification of the time windows for execution of the SpM. 
- Service Method (SvM): conventional service methods. The SvM executions are triggered by service request messages from clients.

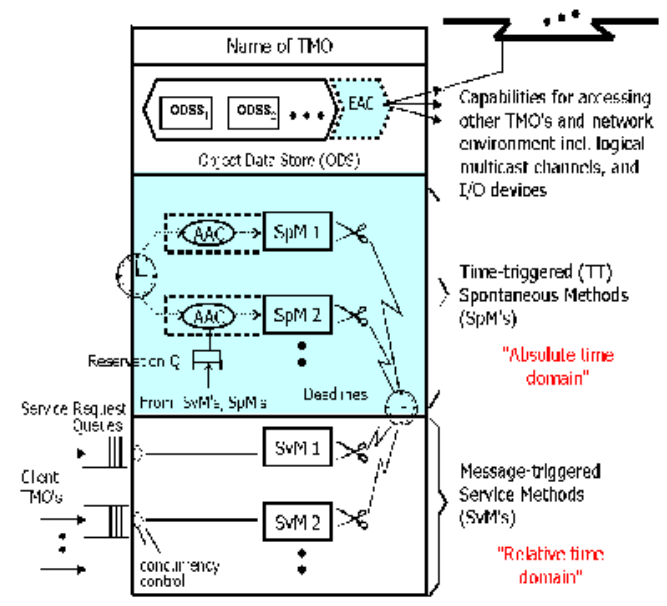

Fig. 1. Structure of TMO (adapted from [5])

\subsection{Feasibility of TMO for Modeling and Implementing Medical Image Processing System}

Fundamental features provided in the TMO programming scheme can enable efficient programming of complex distributed medical image processing system for telemedicine applications. Development of a telemedicine service system requires functionalities of a distributed medical image processing and a medical image processing service. Also, the concept of a service object and provision of high-level API can facilitate the development of a telemedicine system.

First, the TMO scheme provides a sound foundation for programming and executing distributed objects and also the scheme provides various support middleware such as TMOSM/Linux, KelixRT, TMOSM, etc. These features enable a developer to easily design and implement a telemedicine system. Second, the clear separation between SpMs and SvMs, and the BCC rule allow the use of SpM, the time-triggered spontaneous methods, as a means for periodic acquisition of medical signal.

\subsection{DICOM}

The DICOM standard is a specification that describes a means of formatting and exchanging actual image data and associated information such as image calibration and patient's information. The standard applies to the operation of the interface that is used to transfer data in and out of an imaging device such as CT, MRI, X-ray, PET, SPECT, and Ultra-sound [8-10].

Figure 2 shows format of DICOM. DICOM file is a series of "data elements" each of which contains a piece of information. 


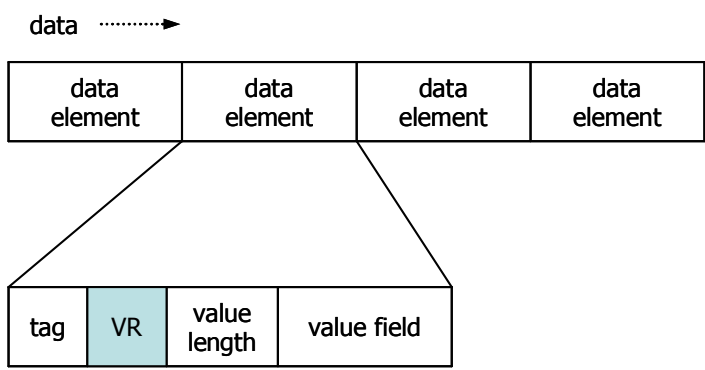

Fig. 2. Format of DICOM
- Data element tag: it contains a piece of information. Each element is described by an "element name" consisting of a pair of 16 bit unsigned integers ("group number", "data element number").

- VR (value representation): it is a field that is a 16 bit unsigned character that describes a type of data and a type of data element value.

- Value length: it is a length field that is a 32 bit unsigned even integer that describes the number of bytes from the end of the length field to the beginning of the next data element.

\section{Design of a Medical Image Processing System Based on the TMO Model}

\subsection{KoMIPS Overview}

KoMIPS is a PC-based cost-effective system that is capable of acquiring, storing, analyzing, and processing medical images. KoMIPS is a result of joint work with a Samsung Medical Center team (department of nuclear medicine) and initial objective of KoMIPS is to facilitate to detect a breast cancer. Figure 3 briefly shows how KoMIPS obtains a medical image. After injection of a radioisotope to a patient, KoMIPS acquires signals using a small gamma camera. Then it constructs, displays, processes, and analyzes an image in real-time. It should be noted that KoMIPS can deal with various types of images although current KoMIPS uses a gamma camera as its scanning device. The gamma camera can be replaced with other scanning devices such as CT (Computer Tomography), MRI (Magnetic Resonance Imaging), SPECT (Single Photon Emission Computerized Tomography), PET (Positron Emission Tomography), etc.

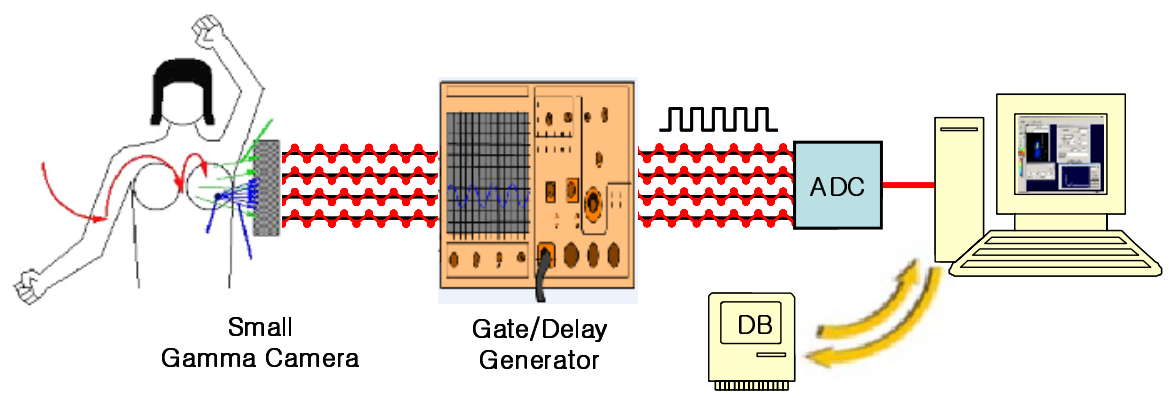

Fig. 3. Overview of KoMIPS 


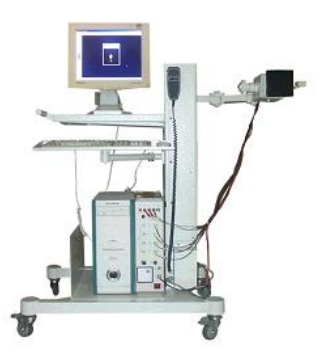

(a) A small gamma camera System

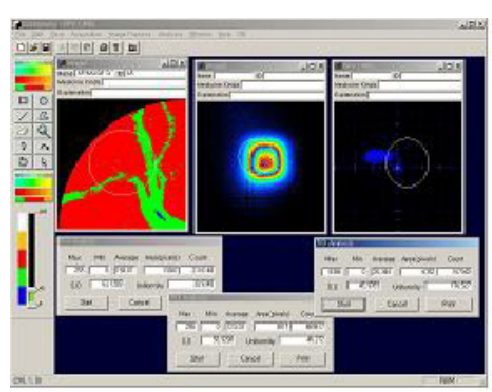

(b) snapshot of KoMIPS

Fig. 4. KoMIPS system

KoMIP can not only load various types of images but also construct images in realtime. Also, KoMIPS converts an acquired raw image into a DICOM image using DICOM encode module and then, stores the image to a database. The Figure 4(a) shows a small gamma camera to acquire a medical image and Figure 4(b) shows a snapshot of image processing in KoMIPS. Several doctors at the Samsung Medical Center have used our KoMIPS prototype extensively and expressed the usefulness of KoMIPS.

\subsection{System Architecture}

With advent of the ubiquitous computing paradigm, research on a telemedicine system is vigorously being progressed. However, research on a ubiquitous telemedicine system is in its beginning stage. Especially, a telemedicine application requiring real-time operations is not being considered yet. In this section, we designed a TMO based medical image processing system, named u-KoMIPS (ubiquitous KoMIPS) running on

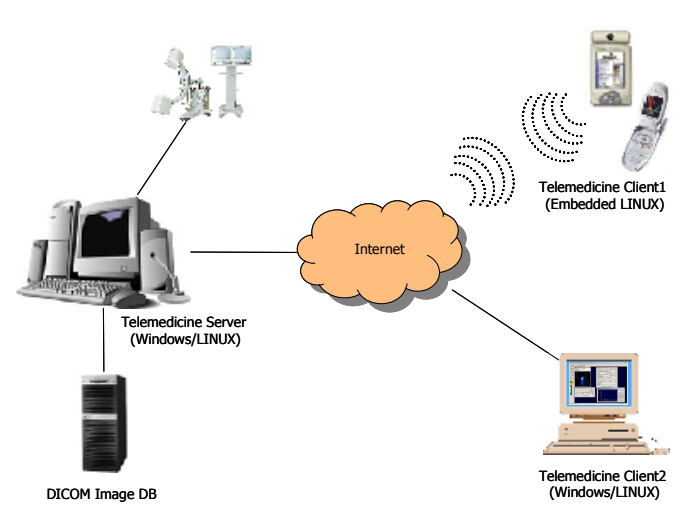

Fig. 5. TMO based medical image processing service TMOSM/Linux. TMOSM/Linux is a middleware supporting real-time operations and execution of TMO structured programs.

Figure 5 shows a service environment of u-KoMIPS. We installed a (embedded) Linux and a TMOSM/Linux that is a middleware supporting TMO. A client such as desktop, PDA, and mobile phone requests an image or an image processing service of server. A (embedded) Linux, a real-time OS, is enable of rapidly, quickly and seamless information processing. 


\subsection{Modeling of a TMO Based Medical Image Processing System}

In this section, we present design of the u-KoMIPS. In the u-KoMIPS, the following objects (TMOs) are used:

- MIAcquisition TMO: an object to acquire a medical image from various medical image scanners such as MRI, CT, SPECT, etc.

- MIProcess TMO: an object to process a medical image.

- MIAnalysis TMO: an object to analyze a medical image.

- MIDicom TMO: an object to convert an acquired medical image into a DICOM image.

- MIDisplay TMO: an object to display DICOM/Raw images on mobile information devices such as a PDA, a cellular phone, a HPC, etc.

- MISManager TMO: an object to overall manage requirement of MIService TMO. MIService TMO request MISManager TMO of a DICOM image or image processing service. Then, MISManager TMO requests to a corresponding TMOs.

- MIService TMO: an object to request an image or image processing service to a corresponding server.

Figure 6 presents the relationship among the above mentioned TMOs. SpMs in each TMO perform the roles assigned to the TMO. Interactions among TMOs are

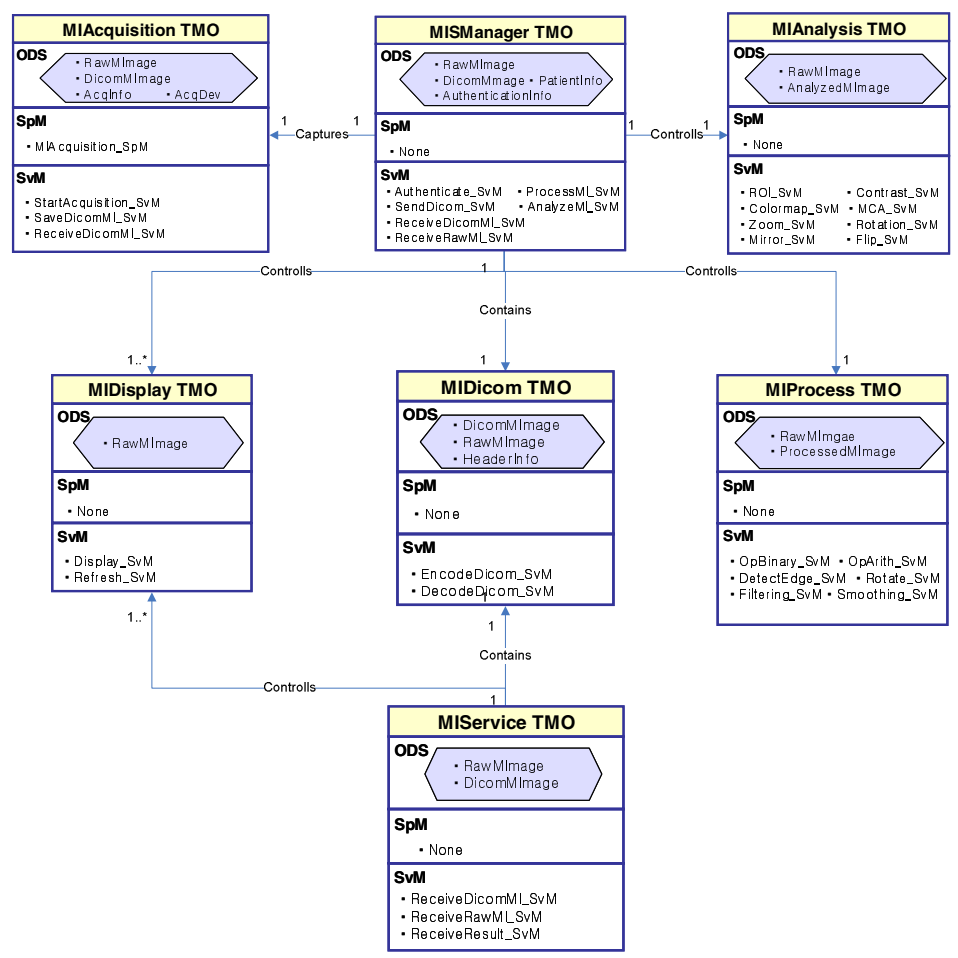

Fig. 6. TMO class diagram for a medical image processing system 
occurred through SvMs and EAC. With MIAcquisition TMO, we explain how the roles of a TMO can be performed by SpMs in that TMO and how a TMO can interact with other TMOs.

MIAcquisition TMO is a service object that inputs/outputs medical image data from various medial image scanners. Among the methods in MIAcquisition TMO, MIAcquisition_SpM acquires raw image data from medical image scanners periodically and saves into a corresponding ODS. In the following, we describe the methods in MIAcquisition TMO in detail.

- MIAcquisition_SpM: MIAcquisition_SpM is automatically activated by AAC allocated dynamically in runtime, periodically acquires image signals from medical image scanner and saves them into ODS. At this point, depending on a medical image scanner, various image composition algorithms are selectively applied. Also MIAcquisition_SpM notifies an acquisition status to MISManager TMO. Specification of temporal conditions in AAC for MIAcquisition_SpM at design time so as follows:

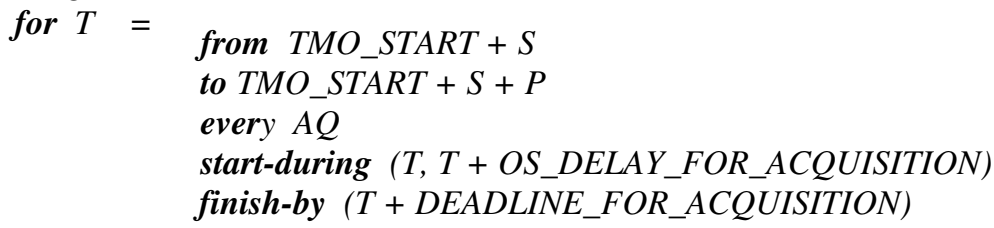

The $\mathrm{S}$ denotes start time that is the time to acquire signal from medical image scanner, and $\mathrm{P}$ denotes stop time that is an end of an acquisition time. The AQ denotes the time that is request time to compose a medical image, and $\mathrm{D}$ denotes deadline time of signal processing. A SpM with the above AAC starts at the time TMO_START $+\mathrm{S}$ and is executed until the time TMO_START $+\mathrm{S}+\mathrm{P}$ with the period AQ. Also, the SpM must start between $\mathrm{T}$ and $\mathrm{T}+$ OS_DELAY_FOR_ACQUISITION and complete its task within $\mathrm{T}+$ DEADLINE_FOR_ACQUISITION. T is a time variable and TMO_START refers to the start time of the TMO execution engine. OS_DELAY_FOR_ACQUISITION is the time spent by OS for activating a SpM.

- StartAcquisition_SvM: a method that is called by MISManager TMO in case of receiving request of an image acquisition from client.

- SaveDicomMI_SvM: a method that is called in case of saving a DICOM image to medical image database. SaveDicomMI_SvM reads a DICOM image data from ODS and saves it DB.

- ReceiveDicomMI_SvM: a method that is called by MIDicom TMO in case of trying to send a DICOM image. ReceiveDicomMI-SvM receives a DICOM image data from MIDicom TMO and writes it to ODS.

\section{A Prototype of u-KoMIPS}

In this section, we present a prototype of the u-KoMIPS. In the prototype, a small gamma camera, a medical image device, is used to acquire a medical image and is applied anger logic algorithm to compose image. 


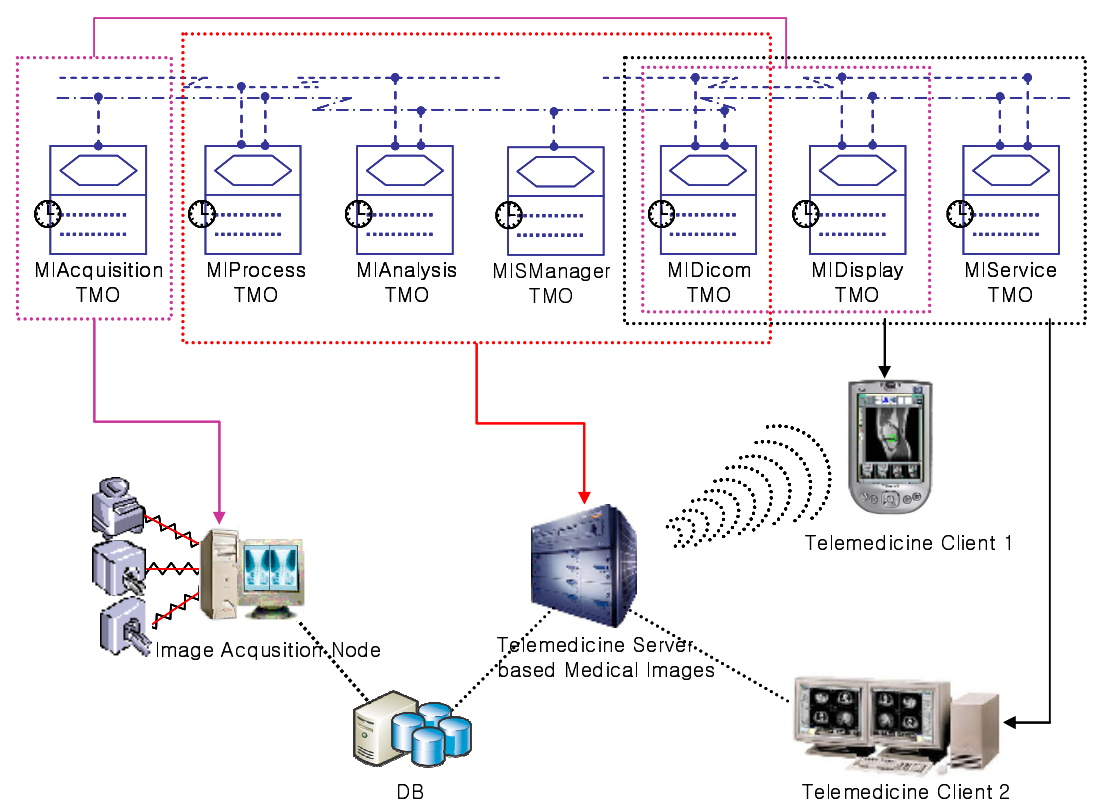

Fig. 7. Design architecture of u-KoMIPS

As depicted in Figure 7, the image acquisition node that acquires image signals from various medical image scanners contains MIAcquisition TMO, MIDicom TMO, and MIDisplay TMO. The Telemedicine server is composed of MIProcess TMO, MIAnalysis TMO, MISManager TMO, and MIDicom TMO. Also, the client is composed of MIDicom TMO, MIDisplay TMO, and MIService TMO. Client requests a medical image or image processing service of telemedicine server with MIService TMO. Then, MISManager TMO received request from client responses it.

Development environment of the u-KoMIPS as follows:

- Operating System : Linux/Embedded Linux

- Middleware : TMOSM/Linux

- ADC: DAQ Board in National Instrument

- Libraries : Nidaq32.lib, Nidex32.lib

- Development Tool : GTK+, QT

- Database : mySQL

Figure 8 shows a GUI of the DICOM Image Viewer, a part of the u-KoMIPS. As depicted in Figure 8, frequently used functions are provided in a form of ICON. Also, at image loading time, the system analyzes the DICOM v3.0 header and displays the header information with image. In addition, the viewer has an image processing tool bar that can be docked [11]. Figure 8(a) shows the server-side GUI that displays DICOM images such as MRI, CT, Gamma, etc. and analyzes images using color map, ROI, and contrast functions. Figure 8(b) shows transfer of an image in a DICOM image database to client. Figure 8(c) shows the client-side GUI. We have implemented server-side and client-side GUIs using QT. 


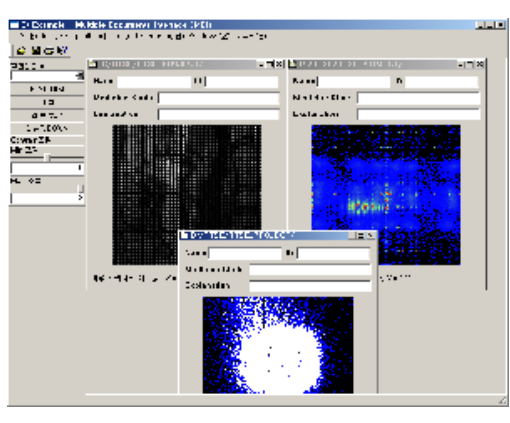

(a) Server-side GUI

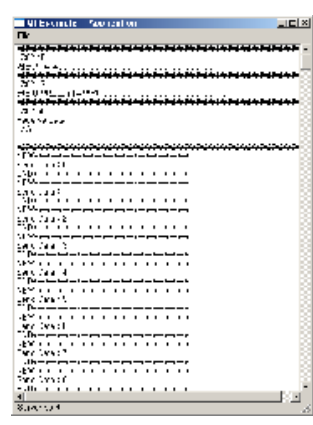

(b) Image transfer

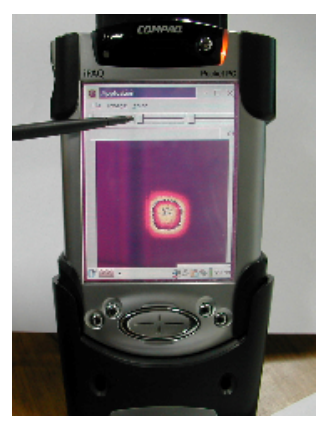

(c) Client

Fig. 8. Prototype of u-KoMIPS

Functions provided with the prototype can be summarized as follows:

- Image acquisition: obtaining raw image data from various sources.

- DICOM encoding/decoding: all images are converted to the corresponding DICOM v3.0 images.

- ROI analysis/copy/paste/save: once ROI is set, then ROI can be copied, pasted, analyzed quantitatively, and saved for future use, e.g., applying saved ROI to same or different images.

- MCA (Multi-Channel Analyzer): for each input channel, the energy (in fact, voltage) of each signal is measured. With signals from all channels, an energy spectrum image can be constructed. This function can improve image analysis capability by removing unnecessary signals and noises prior to the construction of an energy spectrum image.

- Color map control

- Contrast control

- Image processing

- Flip/mirror

- Zoom in/out

- Rotation $\left(0^{\circ}\right.$ to $\left.360^{\circ}\right)$

- Arithmetic/binary operation: image pre-processing such as add, subtract, multiply, divide, AND, OR, and XOR with two images.

\section{Conclusions}

In this paper, we present the design of medical image processing system based on the TMO model for telemedicine applications and implemented a prototype running on PCs and PDAs under the (Embedded) Linux OS. The prototype provides various functions of image processing, analysis, transfer, archive and acquisition. The initial version of our system is named KoMIPS that is a result of joint work with a team in the department of nuclear medicine of the Samsung Medical Center. The current system, named u-KoMIPS, is an extension of KoMIPS of which purpose is to run on a ubiquitous environment. 
Our system provides a way to easily develop telemedicine applications not only in a stand-alone system but also in a ubiquitous/distributed environment. Also, our system can enable mobile clients such as PDA to be used in diagnosing a medical image anytime anywhere.

Acknowledgement. This research was supported by the MIC (Ministry of Information and Communication), Korea, under the University ITRC (Information Technology Research Center) support program supervised by the IITA (Institute of Information Technology Assessment).

\section{References}

1. B. Woodward, R.S.H. Istepanian and C.I. Richards : Design of a Telemedicine System Using a Mobile Telephone, IEEE Trans. On Inf. Tech. in Biomed., Vol. 5, No. 1, March 2001

2. Negel H. Lovell, F. Magrabi, B.G. Celler, K. Huynh and H. Garsden : Web-based Acquisition, Storage, and Retrieval of Biomedical Signals, IEEE Engineering in Medicine and Biology, May/June 2001, pp.38-44

3. J.K. Pollard, S. Rohman and M.E. Fry : A Web-Based Mobile Medical Monitoring System, International Workshop on Intelligent Data Acquisition and Advanced Computing Systems, Technology and Applications, 1-4 July 2001, Foros, Ukraine, pp. 32-35

4. Shawn, et al, "Design and implementation of an Internet-based medical image viewing system", The journal of Systems and Software 66, (2003) 167-181

5. Kim, K.H.: APIs for Real-Time Distributed Object Programming. IEEE computer, (2000) 72-80

6. Kim, K.H., Ishida, M., and Liu, J.: An Efficient Middleware Architecture Supporting Time-Triggered Message-Triggered Objects and an NT-based Implementation. ISORC, (1999) 54-63

7. Kim, H.J., Park, S.H., Kim, J.G., and Kim, M.H.: TMO-Linux: A Linux-based Real-time Operating System Supporting Execution of TMOs, ISORC, (2002)

8. NEMA Standards Publications PS 3.x, "Digital Imaging and Communications in Medicine (DICOM)", National Electrical manufacturers Association, 1998

9. S.C. Horii, et al., "DICOM: An Introduction to the Standard", http://www.xray.hmc.psu.edu/dicom/dicom-_intro/index.html, 1997

10. B.A. Levine, et al., "Challenges encountered while implementing a multi-vendor teleradiology network using DICOM 3.0”, Proc. SPIE 3035, 1997, pp. 237-246

11. Randy Crane, A simplified approach to Image Processing, Prentice Hall, 1997 